\title{
Estimulação transcraniana por corrente contínua em fibromialgia: uma revisão sistemática
}

\author{
Transcranial stimulation by continuous current in \\ fibromyalgia: a systematic review
}

\author{
Estimulación transcraneal de corriente continua en \\ fibromialgia: una revisión sistemática
}

\author{
Alisson Alexandre da Silva ${ }^{1}$, Elaine Kakuta ${ }^{1}$, Eduardo Henrique Loreti ${ }^{2}$ \\ 1.Discente do curso de Fisioterapia no Centro Universitário da Grande Dourados (UNIGRAN), Dourados- \\ MS, Brasil. \\ 2.Fisioterapeuta, Docente no curso de Fisioterapia no Centro Universitário da Grande Dourados \\ (UNIGRAN), Dourados-MS, Brasil.
}

\begin{abstract}
Resumo
Introdução. A fibromialgia afeta a saúde física e mental da população e é caracterizada por alterações na atividade cerebral. A estimulação transcraniana por corrente contínua (tDCS) é uma técnica não invasiva capaz de modular a excitabilidade cortical cerebral. Objetivo. Sistematizar e discutir os efeitos da ETCC na melhora da dor em pacientes com fibromialgia quando comparado ou associado à outras técnicas, partindo dos seguintes questionamentos: A eletroestimulação transcraniana por corrente contínua é eficaz para a melhora da dor? Modifica as características clínicas da doença? Método. Foram selecionados artigos publicados entre $20^{15}$ e 2020 das bases de dados Scielo, MEDLINE, Web of Science e PEDro. Resultados. Foram selecionados 9 artigos, dos quais 6 demonstraram resultados positivos na modulação da dor com estímulo anódico em M1, 2 em CPFDL e 1 em CPFDL + C2. Conclusão. A técnica se mostrou eficaz para a modulação da dor em pacientes com fibromialgia. No entanto, são necessários mais estudos com objetivo de definir a quantidade de sessões ideal, intervalos entre as sessões e dosagem.
\end{abstract}

Unitermos. Fibromialgia; estimulação transcraniana por corrente contínua; dor; dor crônica; doenças reumáticas

\begin{abstract}
Introduction. Fibromyalgia affects the physical and mental health of the population and is characterized by changes in brain activity. Transcranial direct current stimulation (tDCS) is a non-invasive technique capable of modulating cerebral cortical excitability. Objective. Systematize and discuss the effects of tDCS on pain improvement in patients with fibromyalgia when compared or associated with other techniques, based on the following questions: Is transcranial direct current electrical stimulation effective for pain relief? Does it modify the clinical characteristics of the disease? Method. Articles published between 2015 and 2020 were selected from the Scielo, MEDLINE, Web of Science and PEDro databases. Results. Nine articles were selected, of which 6 demonstrated positive results in modulating pain with anodic stimulus in M1, 2 in DLPFC and 1 in DLPFC + C2. Conclusion. The technique proved to be effective for modulating pain in patients with fibromyalgia. However, further studies are needed in order to define the ideal number of sessions, intervals between sessions and dosage. Keywords. Fibromyalgia; transcranial direct current stimulation; pain; chronic pain; rheumatic diseases
\end{abstract}

\section{RESUMEN}

Introducción. La fibromialgia afecta la salud física y mental de la población y se caracteriza por cambios en la actividad cerebral. La estimulación de corriente continua transcraneal (tDCS) 
es una técnica non invasiva que es capaz de modular la excitabilidad cortical cerebral. Objetivos. Sistematizar y discutir los efectos de la estimulación transcraneal de corriente continua en la mejora del dolor en pacientes mayores de 18 años en comparación o asociada con otras técnicas. Método. Se han analizados 9 estudios que realizaban intervenciones activa (la aplicación de la tDCS junto con cualquier tipo de terapia), así como intervenciones sham (tDCS inactiva). Los estudios se publicaron entre los años de 2015 a 2020). En el análisis se revisaron las bases de datos PubMed,Scielo, Medline, WebScience e PEDro. Resultados. Se obtuvieron un total de 304 pacientes, 289 mujeres y 15 hombres. Acerca de las regiones electro estimuladas, el $66,66 \%$ de los estudios se realizó en $M 1$, el $22,22 \%$ en CPFDL y el $11,11 \%$ en CPFDL + C2. Conclusiones. La tDCS activa es una técnica que puede ayudar a mejorar el dolor de las personas con fibromialgia. Tener buenos resultados cuando se asocia con ejercicios aeróbicos y mejora también la depresión, la ansiedad y el estado de ánimo.

Palabras clave: Fibromialgia; estimulación transcraneal de corriente continua; dolor; dolor crónico; enfermedades reumáticas

Trabalho realizado no Centro Universitário da Grande Dourados (UNIGRAN), Dourados-MS, Brasil.

\section{INTRODUÇÃO}

Aproximadamente 30 a $40 \%$ da população mundial sofre com dor. No Brasil, poucos estudos de prevalência são feitos em âmbito nacional, porém em Salvador há uma prevalência de $41,4 \%$ de pessoas que sofrem com dor crônica ${ }^{1}$.

Dentre as doenças que englobam a dor crônica podemos citar a fibromialgia, síndrome caracterizada por alterações nas redes funcionais, química e estruturais do cérebro encarregadas por processar áreas sensoriais e de humor $^{2}$, interferindo nas atividades de vida diária e na qualidade de vida, afetando pessoas de várias idades ${ }^{3,4}$.

A FM causa impactos tanto na saúde física e mental, quanto no relacionamento e carreira profissional da população ${ }^{5}$. Um estudo feito com 118 mulheres com diagnóstico de FM investigou o grau de força muscular, capacidade de andar e desempenho físico associados com 
idade, duração dos sintomas, limitação e índice de massa corporal (IMC). Essas pacientes apresentaram diminuição da capacidade física associada à duração dos sintomas e limitação das atividades de vida diárias ${ }^{6}$.

O uso da Estimulação transcraniana por corrente contínua (ETCC) para o tratamento da dor surgiu com o objetivo de modulação da excitabilidade do córtex motor primário e córtex pré-frontal. A ETCC utilizada em pacientes com fibromialgia a uma intensidade de $2 \mathrm{~mA}$ por 20 minutos durante cinco dias, mostrou melhora da dor por até três semanas após o término do período de estimulação ${ }^{1}$.

Considera-se que a ETCC seja uma técnica segura, de fácil aplicação e eficaz quando comparada às outras técnicas de neuromodulação. Além de possuir aparelhos portáteis e de fácil manuseio dos pacientes, possibilita também associação com técnicas da fisioterapia ${ }^{1}$.

A ETCC possui eficácia para tratamento de sintomas como ansiedade, depressão e distúrbios do sono, sintomas que também englobam o quadro clínico do paciente além da queixa de dor. Dentre as vantagens de sua utilização, a ETCC possui baixo custo, poucos efeitos colaterais, geralmente benignos e alta tolerabilidade do paciente ${ }^{1}$.

Nos últimos anos as publicações de estudos sobre a FM têm aumentado consideravelmente ${ }^{4}$, porém pouco tem sido acrescentado à esta população sobre propostas voltadas a terapias não medicamentosas para analgesia da dor, como a ETCC. Há certa carência também de estudos que relatem sua eficácia na modulação da dor em pacientes com fibromialgia. 
O presente estudo teve como objetivo sistematizar e discutir os efeitos da estimulação transcraniana por corrente contínua na melhora da dor em pacientes acima dos 18 anos quando comparado ou associado à outras técnicas, partindo dos seguintes questionamentos: a eletroestimulação transcraniana por corrente contínua é eficaz para a melhora da dor? Modifica as características clínicas da doença?

\section{MÉTODO}

\section{Bases de dados e critérios de busca}

Foram pesquisados artigos nas bases de dados Scientific Electronic Library Online (Scielo), Medical Literature Analysis and Retrieval System Online (MEDLINE), Web of Science e Physiotherapy Evidence Database (PEDro). A busca pelos artigos aconteceu entre 10 a 20 de março de 2020 usando a combinação dos seguintes descritores, de acordo com o Descritores em Ciências da Saúde (DeCS), nos idiomas inglês e português: "fibromyalgia", transcranial direct current stimulation", "pain", "chronic pain", "rheumatic diseases", "fibromialgia", "estimulação transcraniana por corrente contínua", dor, dor crônica e doenças reumáticas.

\section{Critérios de inclusão e exclusão}

Foram selecionados artigos publicados entre 2015 a 2020; com ETCC aplicada em humanos maiores de 18 anos 
de ambos os sexos com diagnóstico de fibromialgia; ensaios clínicos randomizados com pontuação igual ou superior a 6 na escala PEDro e estudos com utilização de ETCC isolada ou combinada com outra técnica comparado com ETCC sham isolada ou associada a outra técnica. Foram excluídos estudos em modelo animal; estudos que não eram ensaio clínico randomizado ou com pontuação inferior a 6 na escala PEDro; estudos em indivíduos sem diagnóstico de fibromialgia e estudos com resultados não claros ou com erros.

Dois pesquisadores independentes fizeram a busca dos artigos de maneira independente e após realizaram a análise do título e do resumo para identificação dos estudos relevantes. Os estudos selecionados foram analisados na íntegra seguindo os critérios de inclusão. Caso houvesse alguma discordância entre os pesquisadores com relação a algum artigo, um terceiro pesquisador seria acionado para analisar o artigo.

\section{Risco de Viés}

Para diminuir o risco de viés os artigos foram analisados por dois pesquisadores independente, e foi utilizado a escala PEDro para inclusão de artigos com boa qualidade metodológica e para identificar se os resultados dos artigos são facilmente interpretados ou não, sendo utilizado a pontuação 6 como nota de corte. 


\section{RESULTADOS}

No total foram obtidos 60 estudos, destes, 8 foram selecionados por atender os critérios de inclusão (Figura 1), com total de 304 pacientes sendo 289 (95,1\%) do sexo feminino e 15 (4,9\%) do sexo masculino. Desses, obteve-se um $\mathrm{n}$ total de 304 pacientes, 289 do sexo feminino e 15 do sexo masculino. Sobre as regiões eletroestimuladas, 5 estudos utilizaram eletrodos na área do córtex motor primário (M1) e 3 estudos no córtex pré-frontal dorsolateral (CPFDL; Tabela 1).

\section{ETCC anódica sobre M1 associado a exercícios aeróbicos}

Foi incluído apenas um estudo, Mendonça 20167, com total de 45 participantes separados em três grupos, sendo eles ETCC associado a exercícios aeróbicos $(n=15)$, apenas exercícios aeróbicos $(n=15)$ e apenas ETCC $(n=15)$. Os exercícios aeróbicos foram realizados em esteira por um período de 30 minutos por sessão e foram importantes nessa combinação de técnicas devido seu efeito sob as respostas neuroendócrinas no corpo.

Obteve-se bons resultados nos índices de depressão, ansiedade e uma melhora significativa no limiar da dor no grupo de terapia combinada, onde foi associado ETCC + exercícios aeróbicos. Houve um efeito significativo para intensidade da dor, demonstrando que o ETCC + EA foi superior ao EA $(p=0,007)$ e ETCC $(p=0,0056)$. 
Figura 1. Fluxograma dos artigos analisados.

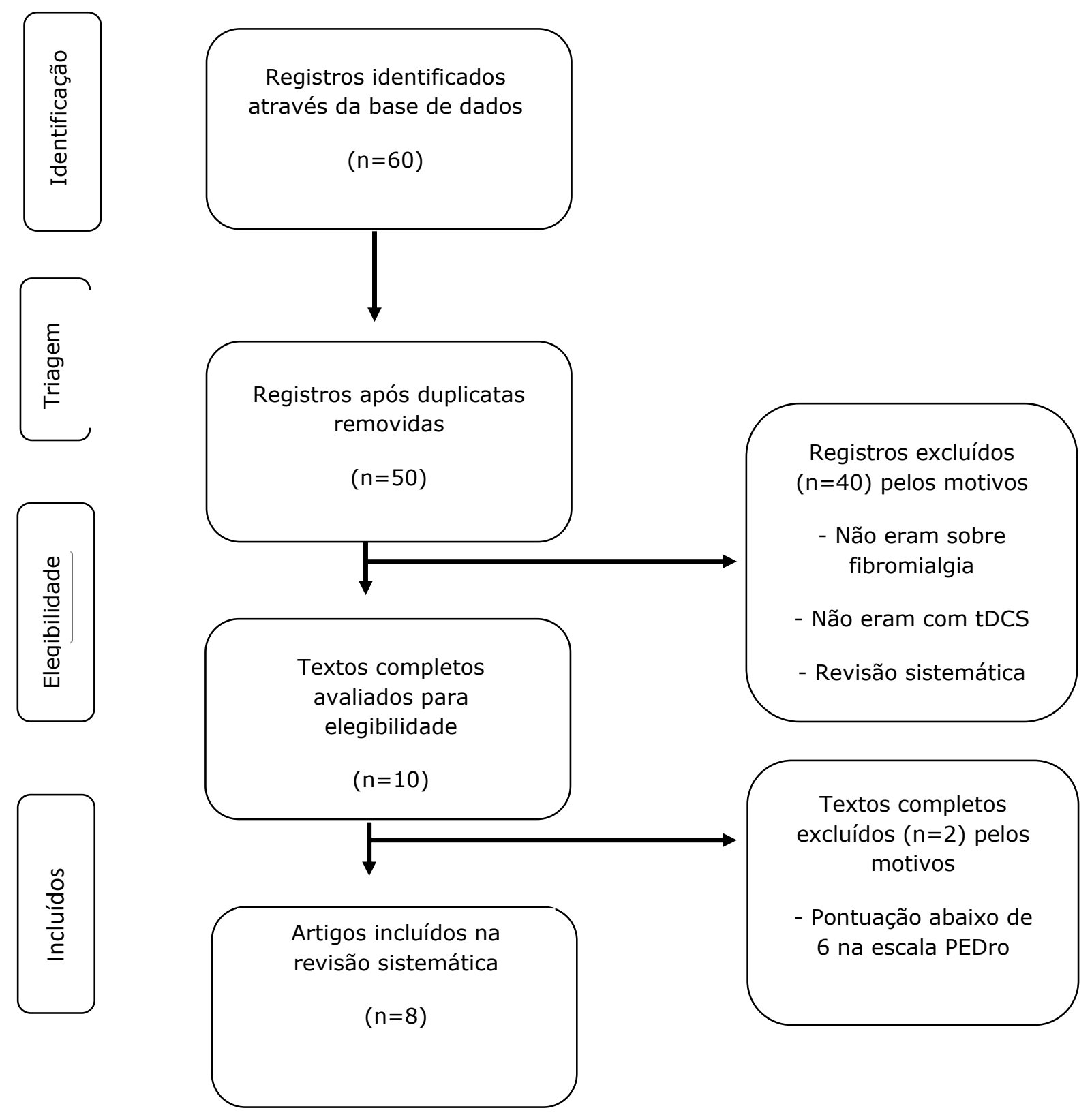


Tabela 1. Artigos referentes aos efeitos da estimulação transcraniana por corrente contínua na melhora da dor.

\begin{tabular}{|c|c|c|c|c|c|c|}
\hline Autor & $\mathbf{n}$ & Local & $\begin{array}{l}\text { Corrent } \\
\text { e }(\mathrm{mA})\end{array}$ & $\begin{array}{l}\text { Duração } \\
\text { Frequência } \\
\text { Sessões }\end{array}$ & Resultados & Escalas \\
\hline $\begin{array}{l}\text { Mendonça } \\
2016^{7}\end{array}$ & $\begin{array}{c}45 \text { pacientes } \\
44 \text { mulheres } \\
1 \text { homem }\end{array}$ & M1 & 2 & $\begin{array}{l}20 \text { min } \\
5 \text { dias } \\
5 \text { sessões }\end{array}$ & $\begin{array}{l}\text { Melhora no } \\
\text { limiar de dor }\end{array}$ & $\begin{array}{l}\text { Escala numérica visual } \\
\text { (VNS), SF36, Inventário } \\
\text { de Beck, limiar de dor a } \\
\text { pressão }\end{array}$ \\
\hline $\begin{array}{l}\text { Santos } \\
2018^{8}\end{array}$ & 40 mulheres & DLPFC & 2 & $\begin{array}{l}20 \text { min } \\
8 \text { dias } \\
8 \text { sessões }\end{array}$ & $\begin{array}{l}\text { A ETCC } \\
\text { combinada } \\
\text { com treinamento } \\
\text { de tarefa } \\
\text { cognitiva } \\
\text { melhorou o } \\
\text { limiar da dor } \\
\text { na FM }\end{array}$ & $\begin{array}{l}\text { Escala visual analógica } \\
\text { (EVA), Inventário de } \\
\text { Beck, Questionário de } \\
\text { Impacto de Fibromialgia } \\
\text { (FIQ), Escala de } \\
\text { pensamentos } \\
\text { catastróficos (BP-PCS), } \\
\text { IDATE, Perfil brasileiro de } \\
\text { dor crônica (B-PCP:S) }\end{array}$ \\
\hline $\begin{array}{l}\text { Silva } \\
2017^{9}\end{array}$ & 40 mulheres & DLPFC & 1 & $\begin{array}{l}20 \text { min } \\
7 \text { dias } \\
2 \text { sessões }\end{array}$ & $\begin{array}{l}\text { A ETCC ativa } \\
\text { aumentou a } \\
\text { tolerância a } \\
\text { dor }\end{array}$ & $\begin{array}{l}\text { Inventário de Beck, EVA, } \\
\text { IDATE }\end{array}$ \\
\hline $\begin{array}{l}\text { Foerster } \\
2015^{10}\end{array}$ & 13 mulheres & M1 & 2 & $\begin{array}{l}20 \text { min } \\
2 \text { semanas } \\
10 \text { sessões }\end{array}$ & $\begin{array}{l}\text { Diminuição } \\
\text { significativa } \\
\text { nos } \\
\text { resultados } \\
\text { clínicos de } \\
\text { dor no grupo } \\
\text { ETCC ativa }\end{array}$ & $\begin{array}{l}\text { EVA, Questionário de dor } \\
\text { de McGill }\end{array}$ \\
\hline $\begin{array}{l}\text { Junior } \\
2015^{11}\end{array}$ & 20 mulheres & M1 & 1 & $\begin{array}{l}20 \text { min } \\
10 \text { semanas } \\
10 \text { sessões }\end{array}$ & $\begin{array}{l}\text { A ETCC foi } \\
\text { eficaz no } \\
\text { controle } \\
\text { terapêutico } \\
\text { da dor }\end{array}$ & $\begin{array}{l}\text { EVA, SF-36, FIQ, } \\
\text { Algiômetro de Fischer } \\
\text { (para mensuração do } \\
\text { nível de dor em pontos } \\
\text { sensíveis) }\end{array}$ \\
\hline $\begin{array}{l}\text { Khedr } \\
2017^{12}\end{array}$ & $\begin{array}{c}36 \text { pacientes } \\
34 \text { mulheres } \\
2 \text { homens }\end{array}$ & M1 & 2 & $\begin{array}{l}20 \text { min } \\
2 \text { semanas } \\
10 \text { sessões }\end{array}$ & $\begin{array}{l}\text { Dez sessões } \\
\text { de } \quad \text { ETCC } \\
\text { podem } \\
\text { induzir alívio } \\
\text { da dor }\end{array}$ & $\begin{array}{l}\text { EVA, Limiar de dor, índice } \\
\text { de dor generalizada } \\
\text { (WPI), Gravidade dos } \\
\text { sintomas da fibromialgia } \\
\text { (SS) }\end{array}$ \\
\hline $\begin{array}{l}\text { Fagerlund } \\
2015^{13}\end{array}$ & $\begin{array}{c}48 \\
\text { participantes } \\
45 \text { mulheres } \\
3 \text { homens }\end{array}$ & M1 & 2 & $\begin{array}{l}20 \text { min } \\
5 \text { dias } \\
5 \text { sessões }\end{array}$ & $\begin{array}{l}\text { Melhora } \\
\text { significativa } \\
\text { da dor }\end{array}$ & $\begin{array}{l}\text { EVA, FIQ, HADS (Hospital } \\
\text { anxiety and depression } \\
\text { scale), Symptom checklist } \\
90, \text { SF36, Escala de } \\
\text { avaliação numérica (NRS) }\end{array}$ \\
\hline To $2017^{14}$ & $\begin{array}{c}42 \text { pacientes } \\
36 \text { mulheres } \\
6 \text { homens }\end{array}$ & $\begin{array}{l}\text { DLPFC e } \\
\quad \text { C2 }\end{array}$ & 1,5 & $\begin{array}{l}20 \text { min } \\
8 \text { semanas } \\
8 \text { sessões }\end{array}$ & $\begin{array}{l}\text { A estimulação } \\
\text { produz } \\
\text { melhora } \\
\text { significativa } \\
\text { da dor }\end{array}$ & $\begin{array}{l}\text { Escala de avaliação } \\
\text { numérica (NRS), Escala } \\
\text { de Catastrofização da dor } \\
\text { (PCS) e Escala de impacto } \\
\text { da fadiga (IFM) }\end{array}$ \\
\hline
\end{tabular}




\section{ETCC anódica sobre M1 vs ETCC sham}

Foram incluídos 4 artigos com total de 137 participantes, sendo 78 no grupo controle e 78 no grupo Sham, desses 8 do sexo masculino e 129 do sexo feminino.

Já no estudo de Foerster 2015 a mesma amostra de pacientes $(n=12)$ participou de estimulação ativa e sham com um intervalo entre as duas de 5 dias ${ }^{10}$. Houve uma diminuição nos resultados clínicos da dor nos pacientes com ETCC ativa.

Junior 2015 analisaram em seu trabalho 20 pacientes (10 sham, 10 ativos) ${ }^{11}$. No grupo que recebeu estimulação ativa observou-se uma redução dos escores do Questionário de Impacto de Fibromialgia $(p=0,0059)$ e da EVA $(p=0,0316)$.

Houve diferença em relação aos valores mensurados de pontos-gatilho e quanto a avaliação do $\operatorname{SF}-36(p<0,05)$. Sendo a ETCC, eficaz no controle terapêutico da dor e promovendo melhora na qualidade de vida dos pacientes com FM.

Khedr 2017 observou que o grupo sham relatou melhora significativa na maioria das escalas de classificação ${ }^{12}$. Isso porque o ETCC inativo produz uma resposta placebo significativa. Houve melhoras em ambos os grupos quanto às escalas de índice de dor. No entanto, a interação entre os grupos na ANOVA bidirecional $(p=0,03$ para escala HAM-D e $p=0,002$ para HAM-A) confirma que a melhora foi maior no grupo experimental quando comparado com o grupo sham. 18 ativo 18 sham. A conclusão foi de que 
10 sessões de etcc ativa induzem alívio da dor e melhora do humor em pacientes com FM.

Fagerlund 2015 dividiram a amostra em 24 pacientes para ETCC ativo e 24 sham $^{13}$. Os resultados sugeriram que a ETCC ativa teve um potencial efeito para induzir alívio da dor em pacientes com FM (redução de $13,6 \%$ no grupo ativo e $1,70 \%$ no grupo sham). Porém, não foi observado nenhum efeito e nenhuma melhoria sobre a saúde mental geral no SF-36, Symptom Checklist 90, HADS e FIQ.

\section{ETCC anódica sobre CPFDL vs ETCC SHAM}

Foram incluídos 3 artigos com total de 122 participantes, sendo 66 no grupo controle e 56 no grupo Sham, desses 6 do sexo masculino e 116 do sexo feminino.

Santos 2018 dividiu sua amostra em 20 pacientes para ETCC ativa e 20 sham, totalizando ao final do estudo 39 pacientes (uma paciente do grupo ETCC ativo abandonou o estudo devido uma lesão na perna) ${ }^{8}$. A ETCC ativa combinada com um treinamento de tarefa cognitiva foi eficiente para melhorar o limiar da dor na FM em comparação com a estimulação sham, além de produzir efeitos em funções cognitivas de memória de curto e longo prazo.

Silva 2017 também dividiu sua amostra em 20 sham e 20 ativo $^{9}$. Porém, 4 indivíduos foram excluídos do estudo porque não entendiam com segurança as instruções ou tinham variabilidade muito extrema nas medidas de dor. Os resultados confirmaram as hipóteses de que o ETCC ativo sobre o CPFDL em comparação com sham, produz mudanças 
significativas no desempenho de uma tarefa cognitiva, nos níveis de atenção e aumenta a tolerância à dor.

Ting 2017 separou a amostra de seu estudo em 15 pacientes em C2, 11 em CPFDL, 16 sham $^{14}$. Todos com 8 sessões duas vezes por semana durante quatro semanas. Os resultados mostraram que as sessões repetidas de ETCC em C2 melhoraram significativamente a dor, mas não a fadiga, em pacientes com FM. Enquanto as sessões em CPFDL melhoraram a dor e a fadiga significativamente.

\section{Eventos adversos}

No estudo de Fagerlund 2015 após cada sessão era aplicado um formulário para que o paciente relatasse qualquer evento adverso, seja dor de cabeça, dor de garganta, coceira, sonolência, mudança de humor, dificuldades de concentração e outros ${ }^{13}$.

Mudança de humor aguda ocorreram com maior frequência após as sessões sham $(6,78 \%)$ do que após sessões ativas $(0,84 \%)$. Os eventos adversos mais frequentes relatados foram vermelhidão da pele ativa/sham $(56,30 \% / 68,64 \%)$, sonolência $(55,46 \% / 50,58 \%)$ e formigação $(53,78 \% / 65,25 \%)$. Não houve eventos adversos graves $^{13}$.

No estudo de Silva 2017 efeitos adversos como formigação, ardor e prurido foram apresentados por 33,33\% dos indivíduos em ETCC sham e 27,5\% em ETCC ativa?. 


\section{DISCUSSÃO}

O mecanismo da dor crônica na FM envolve o desequilíbrio nos circuitos moduladores excitatório (onde há um reforço) e inibitório (há um enfraquecimento) que favorecem e promovem a dor crônica. A desregulação desses sistemas leva a um quadro de sensibilização central que compreende disfunção no sistema modulador descendente da dor e hiperativação de neurônios e da neuroglia ${ }^{16}$.

Através de estudos de imagem pesquisadores obtiveram achados de que os centros corticais e subcorticais que processam aspectos cognitivos e emocionais se comunicam diretamente com os circuitos moduladores da dor, levando a justificativa de como fatores exógenos podem influenciar a dor crônica do indivíduo ${ }^{17}$.

A dor crônica não deve ser considerada uma dor aguda prolongada pois perde sua função biológica e caracteriza-se pela natureza multidimensional, onde além dos fenômenos neurofisiológicos há também os aspectos cognitivos, psicológicos, comportamentais, familiares e sociais ${ }^{18}$.

O mecanismo de ação geral da estimulação se realiza pela modulação do potencial de repouso da membrana plasmática dos neurônios, que possibilita a indução das mudanças de excitabilidade do córtex cerebral através de dois eletrodos (ânodo e cátodo), que quando posicionados, dão origem a um fluxo de corrente elétrica contínua de intensidade pequena mas capaz o suficiente de atingir as regiões específicas do córtex cerebral, modulando de acordo com sua polaridade ${ }^{19}$. 
A estimulação anódica provoca a despolarização da membrana neuronal contribuindo o disparo de potenciais de ação, enquanto a catódica tem efeito contrário, em função da hiperpolarização da membrana neuronal. Enquanto a anódica aumenta a excitabilidade cortical e a catódica diminui, e é essa mudança na excitabilidade cortical que fará com que se realize a modulação da dor ${ }^{20}$.

O tamanho dos eletrodos, seu posicionamento, a duração da estimulação e a densidade da corrente é o que fará com que se obtenha o efeito terapêutico. Alguns autores sugerem e utilizam dois eletrodos ânodo e cátodo de $35 \mathrm{~cm}$, porém, em um estudo onde foi utilizada a técnica de ETCCHD, caracterizada por eletrodos de menor tamanho, houve uma maior densidade de corrente e efeitos de maior duração e com uma estimulação mais concentrada nessas regiões ${ }^{21}$.

A montagem desse estudo foi de 5 eletrodos em formatação 4x1 em que quatro eletrodos de referência foram dispostos ao redor de um eletrodo ativo central, o qual constituía-se de um eletrodo anódico. Os achados foram de que a montagem $4 \times 1$ também teve bons resultados na redução da dor ${ }^{21}$.

Sobre os parâmetros de segurança, é necessário que a ETCC seja aplicada durante 10-20 minutos com uma intensidade de 1 a $2 \mathrm{~mA}$ pois assim o tecido neural não é lesionado ${ }^{11}$.

Nenhum efeito adverso ou colateral grave foi relatado em qualquer dos estudos analisados, com os efeitos relatados (como prurido, sonolência ou formigamento) sendo 
sinais que a estimulação está ativa, causando essas sensações no indivíduo devido à corrente transmitida.

Os efeitos são transitórios, ou seja, não persistem. É extremamente importante investigar o histórico do paciente se ele possui algum diagnóstico ou histórico de condição neurológica ou alguma outra contraindicação ao uso da técnica.

Para a compreender como a ETCC modula a dor, considera-se que existe uma grande rede distribuída de locais no cérebro que é ativada durante o processamento da dor, chamada neuromatriz da dor 22 .

Algumas partes da neuromatriz são superficiais e incluem o córtex sensorial primário (S1), córtex motor primário (M1) e o córtex pré-frontal dorsolateral (CPFDL). Por serem esses locais que contribuem para 0 processamento da dor, normalmente são neles que se realiza a aplicação da eletroestimulação na maioria dos estudos ${ }^{22}$.

Uma revisão sistemática com metanálise de 2020, avaliou a eficácia da ETCC no tratamento da dor em fibromialgia. Quatorze ensaios clínicos foram incluídos e dez eram ensaios controlados. A metanálise de oito ensaios controlados forneceu evidências experimentais de redução da dor quando a ETCC é administrada em comparação com a simulação ${ }^{23}$.

As técnicas de neuromodulação podem ser uma nova forma de tratamento eficaz para condições de dor crônica. Os resultados sugerem que a ETCC ativa aplicada em uma intensidade de $2 \mathrm{~mA}$ para para M1 esquerdo por 20 
minutos/dia por 10 sessões parece ser capaz de diminuir a intensidade da dor na $\mathrm{FM}^{23}$.

Os parâmetros de $2 \mathrm{~mA}$ por 20 minutos por cinco dias consecutivos proporciona não só bons resultados na redução da dor (14-59\%) como também na melhoria da qualidade do sono ${ }^{24}$.

Apenas 40-60\% dos pacientes com dor crônica têm resultado favorável nos tratamentos farmacológicos, sendo a maioria dos tratamentos antidepressivos, opioides e anestésicos tópicos, possuindo eficácia limitada a longo prazo e frequentemente associados a efeitos adversos moderados e em alguns casos graves ${ }^{25}$.

Uma revisão sistemática e metanálise sobre a eficácia da ETCC na fibromialgia, mostrou uma melhora da dor e da função geral relacionada à fibromialgia quando aplicada ETCC anodal sobre o M1 ( $<<0,05)$, no entanto, o limiar da dor à pressão não melhorou $(p>0,05)^{26}$.

Considerando que os efeitos da ETCC são cumulativos e a dificuldade de alguns pacientes e seus cuidadores se deslocarem até as clínicas, houve a necessidade de elaborarse protocolos de tratamento para sua continuidade de forma remota ${ }^{27}$. Essa proposta de tratamento é sugerida porque respeita 0 isolamento social como ocorre nos casos pandêmicos, como é o caso do isolamento social estabelecido devido à pandemia COVID-19.

Em relação à produção científica sobre a ETCC em dor, os dados fornecidos nesta pesquisa mostram que desde a primeira publicação (2006) até a presente data, poucos 
estudos têm abordado o uso de ETCC. O fato de os EUA e o Brasil liderarem com o maior número de publicações pode refletir um pioneiro no uso da técnica, incentivos à pesquisa na área e parcerias entre centros de pesquisa. Estudos têm mostrado a eficácia do uso da ETCC para redução da dor, daí a importância desse tema e dos estudos na área.

\section{CONCLUSÕES}

A técnica ETCC tem se mostrado eficaz para a modulação da dor em pacientes com fibromialgia. No entanto, os números de estudos científicos relevantes sobre seu uso ainda são pequenos, exigindo estudos mais aprofundados e o desenvolvimento de pesquisas em outros centros de pesquisa.

\section{REFERÊNCIAS}

1.Fregni F, Boggio PS, Brunoni AR. Neuromodulação terapêutica. São Paulo: Sarvier Editora de Livros Médicos LTDA, 2012.

2.Cummiford CM, Nascimento TD, Foerster BR, Clauw DJ, Zubieta JK, Harris RE, et al. Changes in resting state functional connectivity after repetitive transcranial direct current stimulation applied to motor córtex in fibromyalgia patients. Arth Res Therap 2016;18:40.

https://doi.org/10.1186/s13075-016-0934-0

3.Villafaina S, Collado-Mateo D, Domínguez-Muñoz FJ, Fuentes-García JP, Gusi N. Impact of adding a cognitive task while performing physical fitness tests in women with fibromyalgia: A cross-sectional descriptive study. Medicine 2018;97:e13791. https://doi.org/10.1097/MD.0000000000013791

4. Häuser W, Fitzcharles MA. Facts and myths pertaining to fibromyalgia. Dialogues Clin Neurosci 2018;20:53-62. https://doi.org/10.31887/DCNS.2018.20.1/whauser

5. Muraleetharan D, Fadich A, Stephenson C, Garney W. Understanding the Impact of Fibromyalgia on Men: Findings From a Nationwide Survey. Am J Men Health 2018;12:952-60.

https://doi.org/10.1177/1557988317753242

6. Larsson A, Palstam A, Bjersing J, Löfgren M, Ernberg M, Kosek E, et al. Controlled, cross-sectional, multi-center study of physical capacity and associated factors in women with fibromyalgia. BMC 
Musculoskeletal Dis 2018;19:121. https://doi.org/10.1186/s12891018-2047-1

7. Mendonça ME, Simis M, Grecco LC, Battistella LR, Baptista AF, Fregni F. Transcranial Direct Current Stimulation Combined with Aerobic Exercise to Optimize Analgesic Responses in Fibromyalgia: A Randomized Placebo-Controlled Clinical Trial. Front Hum Neurosci 2016;10:68. https://doi.org/10.3389/fnhum.2016.00068

8.Santos VS, Zortea M, Alves RL, Naziazeno CCS, Saldanha JS, Carvalho SCR, et al. Cognitive effects of transcranial direct current stimulation combined with working memory training in fibromyalgia: a randomized clinical trial. Sci Rep 2018;8:12477. https://doi.org/10.1038/s41598-018-30127-z

9.Silva AF, Zortea M, Carvalho S, Leite J, Torres ILS, Fregni F, et al. Anodal transcranial direct current stimulation over the left dorsolateral prefrontal cortex modulates attention and pain in fibromyalgia: randomized clinical trial. Sci Rep 2017;7:135. https://doi.org/10.1038/s41598-017-00185-w

10.Foerster BR, Nascimento TD, Deboer M, Bender MA, Rice IC, Truong $\mathrm{DQ}$, et al. Excitatory and inhibitory brain metabolites as targets of motor cortex transcranial direct current stimulation therapy and predictors of its efficacy in fibromyalgia. Arth Rheumatol 2015;67:57681. https://doi.org/10.1002/art.38945

11.Junior LHJ, Costa MDL, Neto LHJ, Ribeiro JPM, Freitas JSN, Teixeira MJ. Estimulação elétrica transcraniana por corrente contínua em fibromialgia: efeitos sobre a dor e a qualidade de vida, avaliados clinicamente e por cintilografia de perfusão cerebral. Rev Dor 2015;16:37-42. https://doi.org/10.5935/1806-0013.20150008

12.Khedr EM, Omran EAH, Ismail NM, El-Hammady DH, Goma SH, Kotb $\mathrm{H}$, et al. Effects of transcranial direct current stimulation on pain, mood and serum endorphin level in the treatment of fibromyalgia: a double blinded randomized clinical trial. Brain Stimul 2017;10:893-901. https://doi.org/10.1016/j.brs.2017.06.006

13. Fagerlund AJ, Hansen O, Aslaksen PM. Transcranial direct current stimulation as a treatment for patients with fibromyalgia. Pain 2015;156:62-71. https://doi.org/10.1016/j.pain.0000000000000006 14.To WT, James E, Ost J, Hart J Jr, De Ridder D, Vanneste S. Differential effects of bifrontal and occipital nerve stimulation on pain and fatigue using transcranial direct current stimulation in fibromyalgia patients. J Neural Transm 2017;124:799-808. https://doi.org/10.1007/s00702-017-1714-y

15. Castillo-Saavedra L, Gebodh N, Bikson M. Clinically Effective Treatment of Fibromyalgia Pain With High-Definition Transcranial Direct Current Stimulation: Phase II Open-Label Dose Optimization. J Pain 2016;17:14-26. https://doi.org/10.1016/j.jpain.2015.09.009

16.Zanette AS. Sistema modulador descendente da dor na fibromialgia: mediadores séricos e efeito da melatonina. Ensaio clínico fase II, Double-dummy, controlado (Tese). Porto Alegre: Faculdade de Medicina da Universidade Federal de Rio Grande do Sul, 2014. 
https://lume.ufrgs.br/bitstream/handle/10183/98468/000922631.pdf ?sequence $=1$ \&isAllowed $=y$

17.Porreca F, Ossipov MH, Gebhart GF. Chronic pain and medullary descending facilitation. Trends Neurosci 2002;25:319-25. https://doi.org/10.1016/s0166-2236(02)02157-4

18. Lamont LA, Tranquilli WJ. Physiology of Pain. Vet Clin North Am Small Anim Pract 2000;30:703-28. https://doi.org/10.1016/s01955616(08)70003-2

19.Gandiga PC, Hummel FC, Cohen LG. Transcranial DC stimulation (tDCS): A tool for doubleblind sham-controlled clinical studies in brain stimulation. Clin Neurophysiol 2006;117:845-50.

https://doi.org/10.1016/j.clinph.2005.12.003

20. Nitsche MA, Paulus W. Excitability changes induced in the human motor córtex by weak transcranial direct current stimulation. J Physiol 2000;527:633-9. https://doi.org/10.1111/j.1469-7793.2000.t01-100633.x

21.Villamar MF, Wivatvongvana $\mathrm{P}$, Patumanond J, Bikson M, Truong $D Q$, Datta $A$, et al. Focal modulation of the primary motor cortex in fibromyalgia using $4 \times 1$-ring high-definition transcranial direct current stimulation (HD-tDCS): immediate and delayed analgesic effects of cathodal and anodal stimulation. J Pain 2013;14:371-83. https://doi.org/10.1016/j.jpain.2012.12.007

22.Vaseghi B, Zoghi M, Jaberzadeh S. A meta-analysis of site-specific effects of cathodal transcranial direct current stimulation on sensory perception and pain. PLoS One 2015;10:e0123873. https://doi.org/10.1371/journal.pone.0123873

23.Lloyd DM, Wittkopf PG, Arendsen LJ, Jones AKP. Is transcranial direct current stimulation (tDCS) effective for the treatment of pain in fibromyalgia? A systematic review and meta-analysis. J Pain 2020;S1526-5900(20)30004-3.

https://doi.org/10.1016/j.jpain.2020.01.003

24.Deus-Yela J, Soler MD, Pelayo-Vergara R, Vidal-Samso J. Transcranial direct current stimulation for the treatment of fibromyalgia: a systematic review. Rev Neurol 2017;65:353-60. https://doi.org/10.33588/rn.6508.2017025

25.Pinto CB, Teixeira CB, Duarte D, Fregni F. Transcranial Direct Current Stimulation as a Therapeutic Tool for Chronic Pain. J ECT 2018;34:e36-50. https://doi.org/10.1097/YCT.0000000000000518 26.Zhu CE, Yu B, Zhang W, Chen WH, Qi Q, Miao Y. Effiectiveness and safety of transcranial direct current stimulation in fibromyalgia: a systematic review and meta-analysis. J Rehabil Med 2017;49:2-9. https://doi.org/10.2340/16501977-2179

27. Charvet LE, Kasschau M, Datta A, Knotkova H, Stevens MC, Alonzo $A$, et al. Remotely-supervised transcranial direct current stimulation (tDCS) for clinical trials: guidelines for technology and protocols. Front Syst Neurosci 2015;9:26. https://doi.org/10.3389/fnsys.2015.00026 